\title{
Complications after breast augmentation with hyaluronic acid: a case report
}

\author{
Emilio Trignano, Manuel Baccari, Nicola Pili, Pietro Luciano Serra, Corrado Rubino \\ Plastic Surgery Unit, Department of Medical, Surgical and Experimental Sciences, Sassari University Hospital Trust, University of Sassari, Sassari, \\ Italy \\ Correspondence to: Dr. Pietro Luciano Serra. Plastic Surgery Unit, Department of Medical, Surgical and Experimental Sciences, Sassari University \\ Hospital Trust, University of Sassari, Sassari, Viale San Pietro 43, 07100 Sassari, Italy. Email: pietro_serra@hotmail.it.
}

\begin{abstract}
Breast augmentation is a very requested procedure in aesthetic surgery. Over time, various techniques of breast augmentation have been described, both through the use of alloplastic materials and autologous tissues: implants, fat grafting, flap surgery, and injectable fillers. According to the international current trend to perform minimally invasive procedure, injectable fillers have progressively increased their uses and notoriety in aesthetic medicine. Among the various available products, hyaluronic acid is likely to be the most popular synthetic filler all over the world. Nowadays many brands are on the market and this paper focuses on Macrolane which is a NASHA-based (stabilized hyaluronic acid of non-animal origin) filler. However, although highly biocompatible, as any material, foreign to our body, also hyaluronic acid fillers undergo a process of degradation and disposal. In our work, we report the first case in literature of migration of Macrolane in an axillary lymph node in a 45 -year-old woman three years after injection for breast augmentation and its related management. The aim of the paper is to discuss and underline further complications and their treatment besides the ones already described in literature in order to provide a deeper knowledge concerning the use and the side effects of injectable fillers in aesthetic medicine.
\end{abstract}

Keywords: Macrolane; breast augmentation; hyaluronic acid; case report

Submitted Apr 08, 2020. Accepted for publication Sep 03, 2020.

doi: $10.21037 / g s-20-448$

View this article at: http://dx.doi.org/10.21037/gs-20-448

\section{Introduction}

Breast augmentation is a very requested and frequent procedure performed by plastic surgeons, in order to achieve the desired breast volume or correct possible asymmetries. Various techniques of breast augmentation have been described: implants, fat grafting, flap surgery, and fillers. According to the current trend to perform minimally invasive procedures, the use of fillers is becoming increasingly popular in cosmetic surgery. Over time, different types of fillers have been used: polyalkylimide gel, polyacrylamide gel, polymethylmethacrylate and hyaluronic acid. The latter is the most commonly used synthetic filler worldwide (1).

It is a glycosaminoglycan biopolymer that the human body is able to synthesize itself. Many hyaluronic acid filler products are available on the market, differentiating from each other for varying levels of hyaluronic acid concentration, degree of cross-linking, particle size, and elastic modulus. As these characteristics change, the indications and sites of injection vary as well (2).

This paper focuses on the use of Macrolane specifically developed for minimally invasive breast volume enhancement.

Macrolane is a NASHA-based (stabilized hyaluronic acid of non-animal origin) medical implant, developed and approved in Europe in 2006 but withdrawn with the market in 2011 because of several reported issues.

Although its approval in Europe, it has never been approved by the U.S. Federal Drug Administration (FDA) and consequently commercialized in the U.S. (1).

The purpose of this paper is to report the first and 


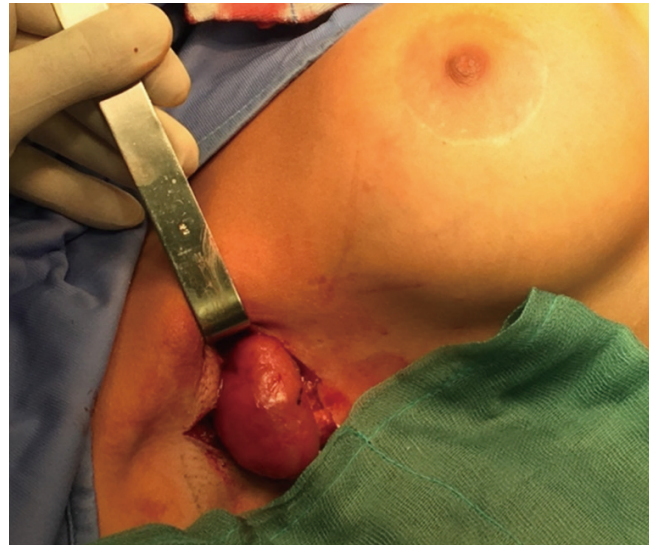

Figure 1 Axillary incision and lymph node exposure.

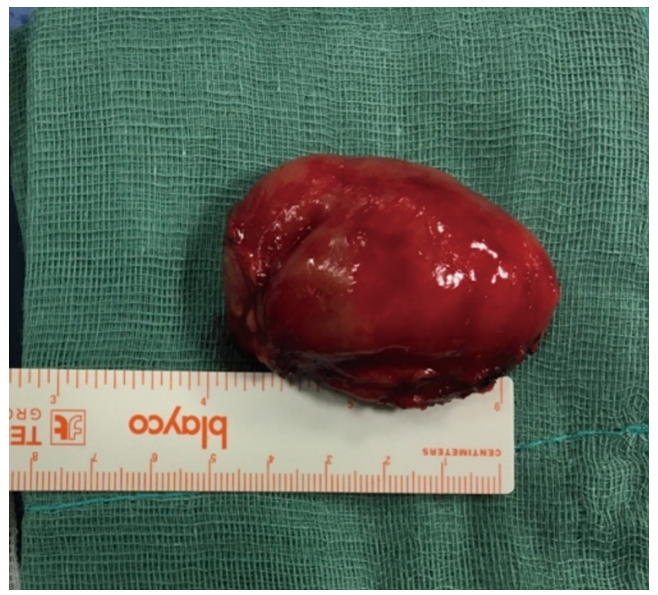

Figure 2 Macrolane lump.

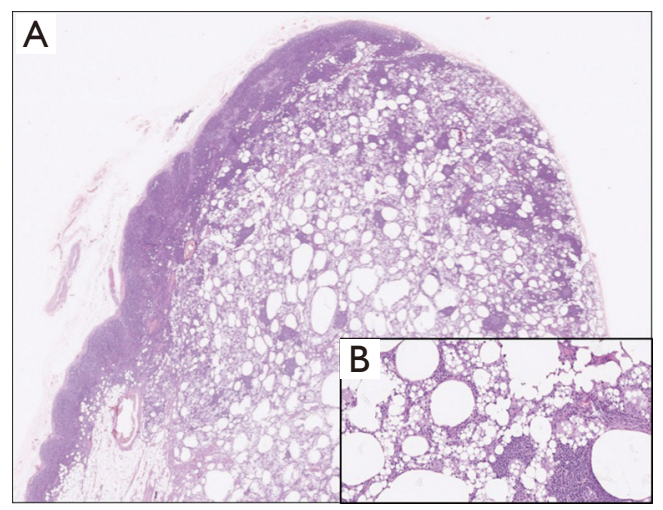

Figure 3 Haematoxylin and eosin pathological image of an axillary lymph node (A, 10x) with a higher magnification (B, 40x) showing accumulation of amorphous material, foamy histiocytes and areas of inflammatory infiltrates. unique case of a woman who was diagnosed with the presence of Macrolane in an axillary lymph node after injection for breast augmentation.

We present the following article in accordance with the CARE reporting checklist (available at http://dx.doi. org/10.21037/gs-20-448).

\section{Case presentation}

A 45-year-old woman requested a plastic surgeon consultation for breast augmentation. After evaluating different treatment options, she opted for temporary breast enlargement with hyaluronic acid; therefore, in the same year, she underwent bilateral breast augmentation with $500 \mathrm{~mL}$ of hyaluronic acid $(250 \mathrm{~mL}$ per breast) (Macrolane; Q-Med AB, Uppsala, Sweden).

After 6 months, the patient presented at our clinic complaining about rapid and asymmetrical volume loss together with lumps formation in both breasts. Locally the breasts appeared reduced in volume, and lumps were both noticeable at inspection and palpable.

After 12 months from Macrolane injections, since the clinical status of the breasts did not improve, the hyaluronic acid lumps were surgically removed and implant-based augmentation mammoplasty was performed through an inferior semi-periareolar incision. In the breast augmentation 345-cc round silicone-filled implants were used (Natrelle Inspira TRM; Allergan, Markham, Ontario, Canada), choosing a sub-muscular placement and using tumescent local anesthesia (3). The postoperative course was uneventful (4).

Three years after surgery, the patient complained about pain in the right axilla associated with the clinical finding of swelling. For this reason, she underwent ultrasound assessment and magnetic resonance imaging of the breasts. One lymph node of the right axilla was swollen $(6 \mathrm{~cm}$ diameter) and presented an altered structure. We opted for surgical removal of the node and the patient was scheduled for surgery 20 days after. The axilla was surgically explored (Figure 1), the node was found and sent for histological examination (Figures 2,3). No complications occurred in the left axilla. The postoperative course was uneventful. The histological examination of the node reported a high concentration of hyaluronic acid, altering the structure of the lymph node.

One month after surgery, the patient did not complain about pain anymore. 
All procedures performed in studies involving human participants were in accordance with the ethical standards of the institutional and/or national research committee(s) and with the Helsinki Declaration (as revised in 2013). Written informed consent was obtained from the patient.

\section{Discussion}

Injectable fillers for breast enhancement allow women and surgeons to decide and obtain the desired breast size. This procedure is performed under local anesthesia, with no hospitalization nor long recovery times. Hyaluronic acid was initially tested as an alternative to collagen for its longer duration of action. Not being a surgical procedure, tissue exposure and trauma are reduced; this leads to a decreased risk of infection and shorter recovery times, so that the patient can rapidly return their normal routine. Moreover, pain management is less demanding and can easily be coped using common NSAIDs.

Macrolane (Q-Med AB, Uppsala, Sweden), is a NASHA (Stabilized Non-Animal Hyaluronic Acid) technology-based hyaluronic acid gel. Its structure includes highly crosslinking in order to elongate its duration in tissues before its absorption (1). Another particular feature of hyaluronic acid fillers is that the degradation of the product is isovolemic, such that as the product degrades, the remaining hyaluronic acid binds additional water to maintain a constant overall volume (2). Macrolane, which was used initially in other body regions for body shaping and volume restoration, in 2008 obtained authorization for breast use (1) but withdrawn with the market in 2011 because of several reported issues.

According to Chaput et al., four main arguments were found: first, the invasiveness of the procedure leads to inflammation which can increase the risk of cancer; second, nodules, resulted from the injection, can cause an interference with the clinical examination; then, the consequent distortion of instrumental examinations images may result in a delayed diagnosis of breast diseases; also, Macrolane is still a recent material, which radiologists are unfamiliar to, that can mimic breast diseases with unnecessary further investigations or potential overtreatment. Finally, it must be said that screening and early diagnosis in breast cancer are a public health priority (1).

Its use has been described for scar reduction and for the correction of contour deformities following liposuction, breast enhancement, buttock augmentation, calf shaping (5), and also augment nipple projection in patients who underwent breast reconstruction (2).

The procedure usually consists of injecting (under ultrasound control) a variable quantity of Macrolane between the pectoralis muscle and the mammary gland. Although, according to the study by Yamaguchi et al., in $\sim 20 \%$ the material was found in, or posterior to, the pectoralis muscle (6). Also, a large quantity persists in the breast tissue for a long time, stimulating an immune response with the consequent development of capsules which surround and envelop the nodules (7).

It is recognized that the absorption time (although with great individual variability) may be quick in some patients, making the relatively low cost of the procedure vain and frequently forcing women to convert the nature of the augmentation with the use of implants (8). A postoperative outcome analysis showed that the Macrolane was present in $78 \%$ of breasts at three months, $57 \%$ of breasts at six months, and only $34 \%$ of breasts at 12 months (5).

Another research conducted by Yamaguchi showed that an overall $79 \%$ of patients saw their breasts improved, much improved, or very much improved six months after treatment, while only a $48 \%$ of them had the same opinion after 12 months (6). Currently, ultrasound, mammography and magnetic resonance imaging, are considered to be the gold standard in diagnosis of breast disease. Although all of them have been described, there is still a lack of data available regarding the use of these techniques in the presence of augmentation mammoplasty performed with hyaluronic acid. Ultrasound is of undeniable value in the diagnosis of augmentation mammoplasty especially in the assessment of breast implants integrity (9). On ultrasound, the Macrolane nodules appears with hypo-anaechogenic cyst-like features. On mammography, the nodules show as gross lobulated radiopacities with polycyclic contours (10). CT and MRI may be useful for further examinations (11). It has been demonstrated that even after more than four years, magnetic resonance imaging and ultrasound can show small amounts of Macrolane in some patients similar to permanent implants; as a consequence the presence of Macrolane gel may interfere with the interpretation of mammography (12).

In the last years, in order to produce an injectable filler with ideal characteristics, different biologic and nonbiologic materials have been studied and commercialized, but none of them has appeared free from risks or side effects. Inevitably, some patients develops complications (11). Complications with Macrolane injections are related to dislocation and infections (13). When Macrolane is 
dislocated, subcutaneous nodules outside of the range of injection can be identified. Although hyaluronic acid is believed to degrade slowly (14), following the same mechanisms of endogenous hyaluronic acid degradation, sometimes it forms subcutaneous nodules, deforming the skin surface. The presence of such nodules not only reduces aesthetic outcome but could interfere with the interpretation of a mammogram, often hindering the early diagnosis of breast cancer (7). Moreover, degraded Macrolane can diffuse into the gland and more likely exit the breasts through the lymphatic system (14). Different authors questioned the diffusion of the product and the risk of complications related $(14,15)$, and in the end, Macrolane was banned for breast augmentation in Europe in 2011 (1).

Macrolane, as every filler, must be injected under aseptic conditions to ensure sterility, nevertheless cases of infections are reported. This risk should always be considered and explained to the patient, and surgeons should sensitize about the importance of returning to the clinic early if symptoms such as pain, redness, and swelling occur. In these cases early treatment is required. If the infection occurs only in one breast, after an antibiotic treatment is often necessary to remove the hyaluronic acid, and consequently a difference in the shape and volume of each breast appears to be evident (13). Since many patients undergo to implant breast augmentation due to loss of volume after Macrolane degradation, as McCleave et al. (16), we strongly believe that the breast implant should be positioned in a Macrolane-free pocket in order to avoid an increased risk of infection of the prosthesis (4).

The removal of hyaluronic acid and the inserting of the implant can be performed in the same surgical time, paying particular attention to potential residual filler which could be reabsorbed over time, reducing the volume of the breast. This eventuality should be discussed with patients (15).

In our case report, even though no major complications occurred, we wanted to point out the unique event of the displacement and accumulation of the hyaluronic acid in an axillary lymph node. Hyaluronidase injection is used to degrade hyaluronic acid when it forms subcutaneous nodules (13), but when a lymph node is affected, this treatment may not be appropriate. We noticed that this complication occurred only unilaterally. We do believe that the complication did not occur in both the right and left axillary lymph nodes because the Macrolane injection was performed mostly in the upper poles in the right breast, while the left one was filled mostly in the medial and inferior poles. The lymphatic drainage has probably merged into another path other than the axilla.

Moreover, in this case report, we wanted to exclude other causes of node swelling. For this reason, we opted for surgical removal of the affected node, followed by the total regression of clinical signs and symptoms in the affected patient. The evidence of hyaluronic acid accumulation in lymph nodes is not yet reported in literature but can be a further demonstration of the unsafe use of Macrolane fillers for breast augmentation.

\section{Acknowledgments}

Funding: None.

\section{Footnote}

Reporting Checklist: The authors have completed the CARE reporting checklist. Available at http://dx.doi.org/10.21037/ gs-20-448

Conflicts of Interest: All authors have completed the ICMJE uniform disclosure form (available at http://dx.doi. org/10.21037/gs-20-448). The authors have no conflicts of interest to declare.

Ethical Statement: The authors are accountable for all aspects of the work in ensuring that questions related to the accuracy or integrity of any part of the work are appropriately investigated and resolved. All procedures performed in studies involving human participants were in accordance with the ethical standards of the institutional and/or national research committee(s) and with the Helsinki Declaration (as revised in 2013). Written informed consent was obtained from the patient.

Open Access Statement: This is an Open Access article distributed in accordance with the Creative Commons Attribution-NonCommercial-NoDerivs 4.0 International License (CC BY-NC-ND 4.0), which permits the noncommercial replication and distribution of the article with the strict proviso that no changes or edits are made and the original work is properly cited (including links to both the formal publication through the relevant DOI and the license). See: https://creativecommons.org/licenses/by-nc-nd/4.0/.

\section{References}

1. Chaput B, De Bonnecaze G, Chavoin JP, et al. France 
prohibits the use of macrolane in aesthetic breast augmentation for reasons similar to criticisms of autologous fat grafting to the breast. Aesthetic Plast Surg 2012;36:1000-1.

2. Sue GR, Seither JG, Nguyen DH. Use of hyaluronic acid filler for enhancement of nipple projection following breast reconstruction: An easy and effective technique. JPRAS Open 2019;23:19-25.

3. Bolletta A, Dessy LA, Fiorot L, et al. Sub-muscular Breast Augmentation Using Tumescent Local Anesthesia. Aesthetic Plast Surg 2019;43:7-13.

4. Fallico N, Faenza M, Dessy LA, et al. Augmentation mammaplasty after breast enhancement with macrolane. Plast Reconstr Surg 2014;133:439e-440e.

5. Nahabedian MY. Discussion. Macrolane for breast enhancement: 12-month follow-up. Plast Reconstr Surg 2011;127:861-2.

6. Yamaguchi S, Nagumo Y, Niwa K. Efficacy and safety of Macrolane $\left({ }^{\mathrm{TM}}\right)$ for breast enhancement: a 12-month follow-up study in Asian women. J Plast Surg Hand Surg 2013;47:191-5.

7. McCleave MJ. Is breast augmentation using hyaluronic acid safe? Aesthetic Plast Surg 2010;34:65-8; discussion 69-70.

8. Goisis M, Savoldi A, Guareschi M. Is hyaluronic acid gel a good option for breast augmentation? Aesthetic Plast Surg 2011;35:134-6; author reply 137 .

Cite this article as: Trignano E, Baccari M, Pili N, Serra PL, Rubino C. Complications after breast augmentation with hyaluronic acid: a case report. Gland Surg 2020;9(6):2193-2197. doi: $10.21037 / g s-20-448$
9. McIntosh SA, Horgan K. Augmentation mammoplasty: effect on diagnosis of breast cancer. J Plast Reconstr Aesthet Surg 2008;61:124-9.

10. Becchere MP, Farace F, Dessena L, et al. A case series study on complications after breast augmentation with Macrolane ${ }^{\mathrm{TM}}$. Aesthetic Plast Surg 2013;37:332-5.

11. Wang Z, Li S, Wang L, et al. Polyacrylamide hydrogel injection for breast augmentation: another injectable failure. Med Sci Monit 2012;18:CR399-408.

12. Scaperrotta G, Satchithananda K, Tengvar M, et al. Radiological assessment of the breast following enhancement with Macrolane: Managing the challenges. Eur J Radiol 2017;86:58-62.

13. Ishii H, Sakata K. Complications and management of breast enhancement using hyaluronic acid. Plast Surg (Oakv) 2014;22:171-4.

14. Hedén P, Olenius M, Tengvar M. Macrolane for breast enhancement: 12-month follow-up. Plast Reconstr Surg 2011;127:850-60.

15. Trignano E, Rusciani A, Armenti AF, et al. Augmentation Mammaplasty After Breast Enhancement With Hyaluronic Acid. Aesthet Surg J 2015;35:NP161-8.

16. McCleave MJ, Grover R, Jones BM. Breast enhancement using Macrolane ${ }^{\mathrm{TM}}$ : a report of complications in three patients and a review of this new product. J Plast Reconstr Aesthet Surg 2010;63:2108-11. 